\title{
Assessment of Medium-chain Triglyceride Feeding in Infants with Cystic Fibrosis
}

\author{
MICHAEL GRACEY, * VALERIE BURKE, and CHARLOTTE M. ANDERSON† \\ From the Gastroenterological Research Unit, Royal Children's Hospital Research Foundation, Royal Children's Hospital, \\ Melbourne 3052, Victoria, Australia
}

Impaired growth is a major clinical feature of cystic fibrosis (CF), and correlates well with the degree of impairment of pulmonary function (Sproul and Huang, 1964), though other factors contribute to poor growth. Steatorrhoea occurs in most patients and is reduced, but rarely eliminated, by administration of pancreatic extracts with meals.

Substitution of medium chain triglycerides (MCT) for the long-chain fats contained in a normal diet is beneficial to patients with pancreatic insufficiency (Greenberger, Ruppert, and Tzagournis, 1967; Burke and Anderson, 1967). This is because of differences between the absorption of MCT, which consists of fatty acids with chain lengths of $\mathrm{C}_{8-10}$, and that of long-chain fats (Isselbacher, 1966). Replacement of dietary longchain fats by MCT has been shown to reduce greatly steatorrhoea in patients with CF (Kuo and Huang, 1965; Burke and Anderson, 1967). Kuo and Huang (1965) also reported increased weight gain in 4 infants fed an MCT diet for 6 weeks and 10 children treated for longer periods. However, the results of more prolonged treatment in a larger number of infants with CF have not been reported. Recently a commercial preparation (Triglyde-Mead Johnson), which contains MCT, has become available. This has prompted us to assess the effect of such a diet on growth rates of infants with CF before the clinical course is complicated by established pulmonary and hepatic disease.

\section{Material and Methods}

This study includes all patients with CF presenting in the first year of life since June 1965. All had clinical features of the disease and high sweat sodium and chloride levels, using the method of Anderson and Freeman (1960). The study, which ceased in June 1968 , included 25 patients.

Received December 24, 1968.

«Supported by a Mead Johnson Research Fellowship. †Present address: Institute of Child Health, Birmingham 16.
The diets were chosen by random selection; 11 patients had most of their dietary fat replaced by MCT (Group A), and 14 were given a normal diet (Group B). 5 patients in Group A and 4 in Group B presented with meconium ileus.

All patients have been reviewed regularly as outpatients. Our usual treatment was carried out in both groups, as follows. All patients were given pancreatic extract (Viokase-Viobin Corporation), with feeaings, salt supplements, and intermittent aerosol therapy, as previously described (Phelan et al., 1969). Oral antibiotics were commonly used, and an antibiotic (usually neomycin) was added to the inhalation diluent in appropriate dosage when there was clinical evidence of chest infection. A mucolytic agent (Nacetyl cysteine) was sometimes used.

Analysis of data. Differences between groups were sought by applying two-way analysis of variance to both chest scores and centiles for weight before and after treatment in group A and group B.

Regression lines, constructed by the method of least squares, were used to determine the possible relation between (a) change in chest scores and change in weight centiles; (b) age at entry to the trial and change in weight centiles; and (c) duration of the trial and change in weight centiles.

Student's $t$ test was applied to show whether the calculated slope of each line was significantly different from zero, and if there was a significant difference between the slopes of the lines for A and B.

At the beginning and end of the trial, centiles for weight were recorded on the Stuart centile grid (Stuart et al., 1959), and the severity of pulmonary disease was assessed using the scoring system shown (Table I). The total chest score was obtained by combining the score for pulmonary sigrs and the score for pulmonary symptoms. These values for centiles and 'chest scores' were used in statistical analysis (Table II).

\section{Results}

In all patients treated with MCT the stools became less frequent, bulky, and offensive.

At entry to the trial, group A and B had similar chest scores $(p=0 \cdot 1)$ and weight centiles $(p>0 \cdot 1)$. At the conclusion of the trial, analysis of variance failed to show a significant difference between $A$ 
TABLE I

Assessment of Pulmonary Status of Infants with Cystic Fibrosis

\begin{tabular}{|c|c|c|c|}
\hline \multirow{2}{*}{ Grading } & \multirow{2}{*}{ Points } & \multicolumn{2}{|c|}{ Chest Score } \\
\hline & & Pulmonary Symptoms & Pulmonary Signs \\
\hline Excellent & 4 & $\begin{array}{l}\text { Rarely coughs or } \\
\text { wheezes }\end{array}$ & $\begin{array}{l}\text { No chest retraction; } \\
\text { chest normal on } \\
\text { examination }\end{array}$ \\
\hline Mild & 3 & $\begin{array}{l}\text { Occasional cough } \\
\text { or wheeze }\end{array}$ & $\begin{array}{l}\text { Minimal or mild } \\
\text { chest retraction; } \\
\text { occasional } \\
\text { adventitiae }\end{array}$ \\
\hline Moderate & 2 & $\begin{array}{l}\text { Frequent cough } \\
\text { or wheeze }\end{array}$ & $\begin{array}{l}\text { Moderate chest } \\
\text { retraction; } \\
\text { persistent local- } \\
\text { ized adventitiae }\end{array}$ \\
\hline Severe & 1 & $\begin{array}{l}\text { Persistent cough } \\
\text { or wheeze }\end{array}$ & $\begin{array}{l}\text { Persistent chest } \\
\text { retraction and } \\
\text { persistent wide- } \\
\text { spread adventitiae }\end{array}$ \\
\hline
\end{tabular}

and $B$ for chest scores $(p>0 \cdot 1)$ and for weight centiles $(p>0 \cdot 1)$. It seemed possible that inclusion of patients presenting with meconium ileus might have affected the validity of these results, as appropriate surgical management is usually followed by marked clinical improvement.
We, therefore, compared these particular infants with the remainder in each group at the start and finish of the trial. No difference could be shown for chest scores or weight centiles in either group before or after treatment ( $p>0 \cdot 1$ for each comparison), so that subsequent analysis includes all patients.

In group $\mathrm{A}$ there was a significant difference in weight centiles at the beginning and end of the trial $(p<0.005)$. A similar difference occurred in group $B$, though the $p$ value was greater $(<0.025)$. Chest scores of each group were also compared, and again the difference at the beginning and end was significant ( $p<0.005$ for $A ; p<$ 0.005 for B) (Table III).

The regression line relating change in chest scores and change in weight centiles was significant for each group ( $p<0.001$ for $A ; p<0.005$ for $B)$. Comparison of the two regression lines showed that there was a significant difference in their gradients $(p<0.001)$ (Figure).

Finally, the effect of age at entry to the trial and duration of treatment on change in centiles was evaluated. Regression lines relating age and change in weight centile failed to show a gradient

TABLE II

Centiles for Weight and Chest Scores for Infants with Cystic Fibrosis Treated with MCT (Group A, MCT Treated), and those on Normal Diets (Group B, Controls)

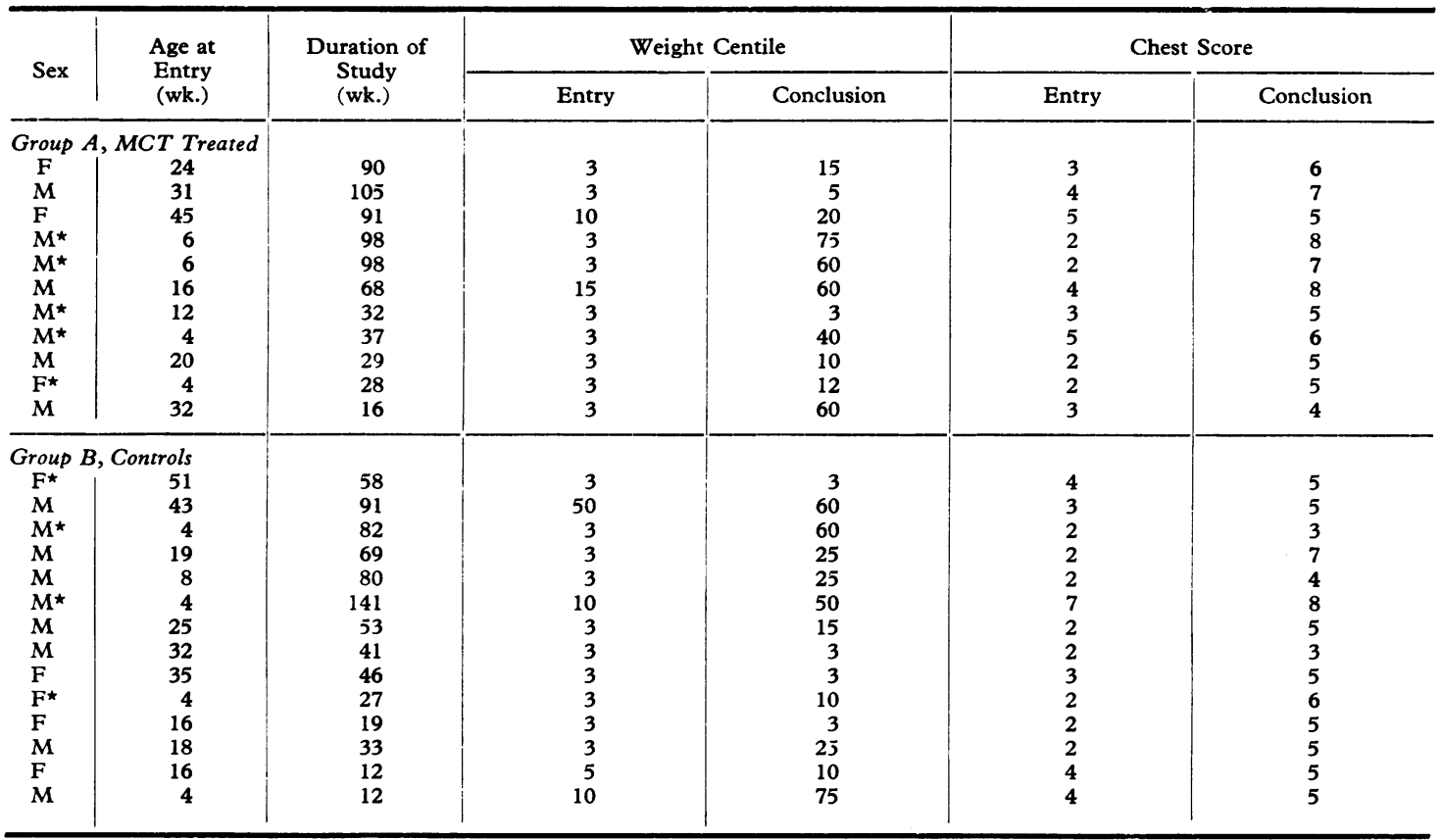

^Presented with meconium ileus. 
significantly different from zero for group A $(p=0 \cdot 1)$ or group B $(p>0.95))$. Similarly, no significant relation between duration of therapy and change in centile was found $(p=0.4$ for $A$; $p=0 \cdot 2$ for $B)$.

\section{Discussion}

Comparative growth studies in small groups of infants are difficult; particularly because the relation of growth rates to time (i.e. growth velocity) is not uniform in the early months of life. For these reasons we have compared centiles for weight, rather than absolute weights, in the two groups of infants under study. Because of the unpredictable clinical course of the disease in any particular patient it was decided to study randomly selected patients within groups rather than to attempt to use 'matched pairs'. We have shown a significant improvement in centiles in both MCT 'treated' and 'control' groups during the period under consideration.

There is no doubt that accelerated growth correlates well with effective treatment of pulmonary disease (Sproul and Huang, 1964). This has been shown in both groups in the present study. However, comparison of regression lines relating change in chest scores to changes in centiles has shown a difference in their gradients which could be explained by improved chest scores in Group A or improved growth velocity in Group B. This differs from the short-term improvement in weight gain by Kuo and Huang (1965) in patients given MCT.

Larger groups of patients with cystic fibrosis must be studied further to establish whether longterm feeding with MCT favourably influences their growth rates over longer periods.

\section{Summary}

The effect of medium-chain triglyceride (MCT) feeding on growth rates of infants with cystic fibrosis (CF) over a period of three years was studied. Diets were randomly selected: 11 patients were given the MCT diet; 14 patients were used as 'controls'. In all patients treated with MCT the stools became less frequent, bulky, and offensive.

Using analysis of variance, no significant difference could be shown between chest scores and weight centiles for the MCT treated and 'control' groups at the end of the trial $(p>0 \cdot 1)$.

Accelerated growth was shown to correlate well with effective treatment of pulmonary disease.

REFERENCES

Anderson, C. M., and Freeman, M. (1960). 'Sweat test' results in normal persons of different ages compared with families of fibrocystic disease of the pancreas. Arch. Dis. Childh., 35, 581 .

\section{TABLE III}

Mean Values for Chest Scores and Weight Centiles before and after Treatment in Group $A$ (MCT Treated) and Group B (Controls)

\begin{tabular}{l|cc|c|c}
\hline & Before Treatment & $\begin{array}{c}\text { After } \\
\text { Treatment }\end{array}$ & \multirow{2}{*}{${ }^{*}$ p Value } \\
\hline \multirow{2}{*}{ Chest Scores } & Group A & 3.0 & 5.7 & $\mathrm{p}<0.005$ \\
& Group B & 2.9 & 5.1 & $\mathrm{p}<0.005$ \\
\hline \multirow{2}{*}{ Weight Centiles } & Group A & 4.7 & 31.9 & $\mathrm{p}<0.005$ \\
& Group B & 7.5 & 26.2 & $\mathrm{p}<0.025$ \\
\hline
\end{tabular}

${ }^{\star} p$ values obtained by analysis of variance.

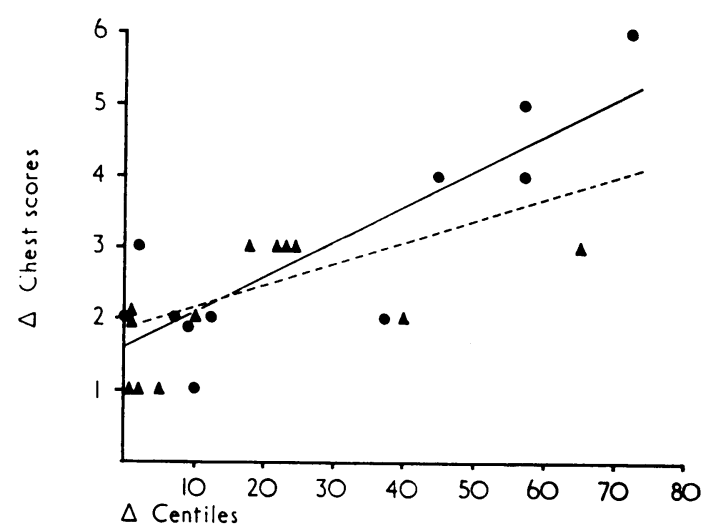

FIG.-Regression lines relating change in chest scores and change in weight centiles, showing a significantly greater slope of the line for Group A (MCT treated; circles, solid line) than for Group $B$ (controls; triangles, dotted line $). \quad(p<0.001$.

Burke, V., and Anderson, C. M. (1967). Experiences with medium chain triglycerides in malabsorptive states in childhood. Aust. paediat. $\mathcal{F} ., 3,135$.

Greenberger, N. J., Ruppert, R. D., and Tzagournis, M. (1967) Use of medium chain triglycerides in malabsorption. Ann. intern. Med., 66, 727.

Isselbacher, K. J. (1966). Biochemical aspects of fat absorption. Gastroenterology, 50, 78.

Kuo, P. T., and Huang, N. N. (1965). The effect of medium chain triglyceride upon fat absorption and plasma lipid and depot fat of children with cystic fibrosis of the pancreas. $\mathcal{f}$. clin. Invest., 44, 1924.

Phelan, P. D., Gracey, M., Williams, H. E., and Anderson, C. M. (1969). Ventilatory function in infants with cystic fibrosis. A physiological assessment of therapy. Arch. Dis. Childh., 44, 393.

Sproul, A., and Huang, N. (1964). Growth patterns in children with cystic fibrosis. F. Pediat., 65, 664.

Stuart, H. C., Reed, R. B., et al. (1959). Description of project (longitudinal studies of child health and development). Pediatrics, 24, 875.

Correspondence to Professor Charlotte M. Anderson, The Institute of Child Health, The Nuffield Building, Francis Road, Birmingham 16. 